\title{
Zur Kenntnis der Taxonomie und Verbreitung der Alpenpflanzen
}

\section{Journal Article}

Author(s):

Landolt, Elias

Publication date:

2006-06

Permanent link:

https://doi.org/10.3929/ethz-b-000022891

Rights / license:

In Copyright - Non-Commercial Use Permitted

Originally published in:

Botanica Helvetica 116(1), https://doi.org/10.1007/s00035-006-0746-y 


\title{
Zur Kenntnis der Taxonomie und Verbreitung der Alpenpflanzen
}

\section{Elias Landolt}

Institut für Integrative Biologie, ETH Zürich, Universitätsstr. 16, CH-8092 Zürich; e-mail: elias.landolt@env.ethz.ch

Manuskript angenommen am 7. März 2006

\begin{abstract}
Landolt E. 2006. Remarks on the present taxonomic and chorological knowledge of the Alpine flora. Bot. Helv. 116: 79-90.

Recently published Floras of the Alps or of parts of the Alps show that our taxonomic and chorological knowledge of the Alpine flora is still incomplete regarding the morphology, delimitation and distribution of many taxa. The situation is demonstrated and discussed by different examples. Compared to Floras of the 20th century only few of the many critical aggregates of taxa have been clarified despite new study methods. A proposal is given to survey and improve our information on Alpine plant taxa.
\end{abstract}

Key words: Alps, cytology, Flora Alpina, distribution, taxonomy.

\section{Einleitung}

Die 2004 publizierte Flora alpina von Aeschimann, Lauber, Moser und Theurillat gibt einen Überblick über den heutigen Stand der Artenkenntnisse der Farn- und Blütenpflanzen des Alpenbogens. Zum ersten Mal in der Geschichte der Erforschung der Alpenflora wurde versucht, alle Taxa der Gefässpflanzen der gesamten Alpen zusammenzustellen. Dazu wurde umfangreiches Datenmaterial als Grundlage verarbeitet und das Wissen von zahlreichen Experten berücksichtigt.

Dieses Werk ist Anlass, den Fortschritt in unserer Kenntnis gegenüber früheren Ausgaben von Land- und Regionalfloren festzustellen und auf allfällige Forschungslücken und Unsicherheiten aufmerksam zu machen. Jedes wissenschaftliche Florenwerk hat das allgemeine Ziel, aufzuzeigen, welche Taxa im untersuchten Gebiet bekannt sind, und wie gut diese morphologisch, ökologisch und chorologisch erfasst werden können. Wie jedes andere Florenwerk spiegelt auch die Flora alpina die Zielsetzung, Betrachtungsweise und Kenntnisse ihrer Autoren wider. Dies beeinflusst die Auswahl und die systematische Einordnung der aufgenommenen Taxa sowie die Art und Genauigkeit der beigegebenen Information. Dabei ist zu berücksichtigen, dass die Flora alpina nach wissenschaftlichen Gesichtspunkten erarbeitet worden ist, sich aber 
auch an Liebhaberbotaniker wendet. Entsprechend wurde eine vereinfachte Darstellung gewählt, welche stellenweise zu Informationsverlusten führt. So wurde beispielsweise auf eine morphologische Beschreibung und eine kritische Betrachtung der Taxa mit Literaturhinweisen verzichtet. Das hat den Nachteil, dass nicht ersichtlich wird, warum einzelne Taxa aus früheren Floren aufgenommen sind und andere nicht.

Die folgenden Ausführungen sollen keine Kritik an diesem neuen Florenwerk sein, sondern stellvertretend für viele neuere Floren aufzeigen, dass trotz langjähriger Tradition in der taxonomischen Bearbeitung der Alpenflora die wissenschaftliche Erforschung der Alpenflora heute keineswegs abgeschlossen ist. Es soll auch dargelegt werden, wie schwierig es ist, die weit gestreute und oft schwierig auffindbare ältere Literatur zu berücksichtigen und $\mathrm{zu}$ gewichten. Es wäre indessen wünschenswert, dass bereits vorhandene Kenntnisse nicht wieder neu erarbeitet werden müssen.

In einem ersten Kapitel werden allgemeine Kriterien für die Berücksichtigung von Taxa diskutiert, und es wird auf das Problem der taxonomischen Bewertung eingegangen. In einem zweiten Kapitel folgen Beispiele, die den Stand der heutigen Kenntnisse erörtern und auf Probleme und Unsicherheiten bei der Auswahl der darzustellenden Taxa hinweisen. Das dritte Kapitel enthält weitere Beispiele, um die Probleme der Kenntnis und Darstellung der Verbreitung aufzuzeigen. Die ökologischen Eigenschaften der einzelnen Taxa werden von mir nicht näher beleuchtet, da sie in fast allen neueren Werken nach unserem heutigen Wissen kompetent und zweckmässig dargeboten werden. Gerade die Flora alpina bietet auf kleinem Raum durch die Verwendung von Symbolen und Schemata viel wichtige Information. Das letzte Kapitel bringt als Folgerungen einige Vorschläge für eine zielgerichtete weitere Erforschung der kritischen Aggregate der Alpenflora.

Die von mir aufgeführten Beispiele betreffen vorwiegend Pflanzengruppen, die mir gut bekannt sind und mit denen ich mich längere Zeit beschäftigt habe. Ich bin überzeugt, dass diese Gruppen repräsentativ für die gesamte Alpenflora sind und dass für viele andere Verwandtschaftskreise ähnliche Verhältnisse gelten.

\section{Allgemeine Kriterien für die Berücksichtigung von Taxa - Taxonomische Bewertung}

Die Alpen sind eine pflanzengeographische Einheit mit einer vielfältigen und doch übersichtlichen Flora und haben eine jahrhundertealte botanische Forschungstradition. Der Fortschritt der floristischen Forschung im Alpenraum wurde in einer Vielzahl regionaler Florenwerke dokumentiert, welche in den vergangenen 200 Jahren immer wieder aufgrund der neuen taxonomischen Kenntnisse bearbeitet und ergänzt wurden. In neuerer Zeit kommen elektronische Datenbanken hinzu (z.B. Moser et al. 2005; www.wsl.ch/ land/products/webflora). Wichtig ist dieser Fortschritt deshalb, weil die Taxonomie die Basis jeder Pflanzenstudie darstellt. Sowohl ökologische, physiologische, zytologische, molekularbiologische, pharmazeutische als auch naturschützerische Studien sind auf eine korrekte Bestimmung und eine eindeutige Bezeichnung der untersuchten Taxa angewiesen. Eine gute Kenntnis der Biologie und Verbreitung jedes Taxons gibt zudem einen Einblick in die Evolution der Arten und ist wichtig, um die Folgen von Veränderungen der Standortbedingungen (Boden, Klima usw.) festzustellen.

Taxa, die sinnvoll in eine Flora oder in andere wissenschaftliche Publikationen aufzunehmen sind, müssen nach meiner Ansicht die folgenden zwei Vorgaben erfüllen: sie sollen 
- erkennbare morphologische Unterschiede aufweisen und

- ökologisch und/oder geographisch eine gewisse Eigenständigkeit zeigen.

Dabei spielt es keine Rolle, ob ein Genfluss zwischen den Populationen stattfindet und Übergangsformen häufig auftreten oder nicht. Oftmals lassen sich morphologische Unterschiede kaum in allseits verständliche Worte fassen, oder die Taxa weisen im Berührungsgebiet breite Übergangszonen auf. Trotzdem sind solche Einheiten klar zu trennen von Gruppierungen charakteristischer Einzelpflanzen, deren Merkmalskombinationen aber innerhalb der Variation von Populationen stehen.

Die Einstufung der behandelten Einheiten als Arten, Kleinarten, Unterarten oder Varietäten wird in verschiedenen Floren unterschiedlich gehandhabt, und es fehlen allgemein anzuwendende Kriterien zur nachvollziehbaren Definition dieser Stufen. Selbst wenn man gewisse Bedingungen für den Artrang aufstellt, etwa keine oder nur geringe Bastardierungsmöglichkeit, kein Genfluss usw., ist unser Wissen über das Verhalten nah verwandter Taxa meist zu gering, um eine Definition konsequent durchziehen zu können.

Als Beispiel solcher Unsicherheiten sei das Aggregat der Scabiosa columbaria L. genannt, dessen zahlreiche Taxa von Afrika bis Südskandinavien im Experiment miteinander fertil bastardieren und in der Natur morphologisch oft breite Übergangszonen bilden (Grossmann 1975). Im Synonymie-Index (Aeschimann und Heitz 2005) sind die meisten Taxa dieser Gruppe als Arten dargestellt. Die südalpin-apenninische Scabiosa portae A. Kerner dagegen ist S. columbaria als Unterart angegliedert. In den Südalpen (z.B. Tessin) sind aber die Pflanzen der mageren Halbtrockenrasen unterer Lagen im Allgemeinen einheitlich und von der dort nicht vorkommenden S. columbaria s.str. morphologisch klar getrennt. Wird dieses Taxon als Unterart eingestuft, müssten konsequenterweise alle anderen Taxa der Gruppe $S$. columbaria gleich behandelt werden. Einzig die gelb blühenden Taxa zeigen gegenüber den blau und rot blühenden weniger breite Übergangszonen, wobei aber auch hier Bastardschwärme auftreten können (Landolt unveröffentlicht).

Wo soll da die Grenze zwischen Art, Unterart und Varietät gezogen werden? Bei unseren einheimischen sommergrünen Eichen kommen Bastarde und Genfluss vor. Die verschiedenen Taxa werden trotzdem fast immer als Arten bezeichnet. Auch die vielen Kleinarten von sich apomiktisch fortpflanzenden Aggregaten erhalten heute in der Regel den Status einer Art, während morphologisch besser umschriebene sexuelle Taxa, die miteinander bastardieren, sich oft mit der Stufe Unterart oder Varietät begnügen müssen. Um solchen Inkonsequenzen auszuweichen, ziehe ich vor, jedes morphologisch, standörtlich und geographisch gut charakterisierbare Taxon mit einem binären Namen zu versehen. Die nahe Verwandtschaft der Taxa kann durch Bildung von Aggregaten oder Artengruppen hervorgehoben werden.

Die ungleiche Beurteilung der taxonomischen Wertigkeit wiegt an und für sich nicht schwer. Es sollte hier lediglich gezeigt werden, wie eine allseits befriedigende Lösung in dieser Frage kaum möglich ist.

\section{Stand der taxonomischen Kenntnisse}

\section{Zunehmende Artenzahlen}

Vergleichen wir die Artenzahlen in verschiedenen Floren oder Florenlisten, so stellen wir fest, dass die Zahl in den letzten Jahren zugenommen hat. So zeigt etwa Kre- 
mer (2004) anhand von Artenlisten aus Deutschland, wie rasch diese Entwicklung stattfindet: 1988 (2490 Taxa), 1996 (3319 Taxa), 1998 (3661 Taxa) und 2000 (3914 Taxa). Auch wenn die angeführten Listen nicht durchwegs vergleichbar sind, ist doch die Tendenz augenfällig. Die höheren Zahlen beruhen nur zu einem kleineren Teil auf der besseren wissenschaftlichen Erforschung der Gesamtflora. Der Zuwachs besteht vorwiegend aus Neophyten, die stets neu einwandern und mit der Erwärmung zugenommen haben; aber auch einige apomiktische Gruppen werden heute vermehrt in Kleinarten unterteilt und teilweise in die Floren aufgenommen.

Von den Neophyten sind erstaunlich viele neue Taxa in der Flora alpina enthalten. Dies zeigt, dass die Einschleppung von Neuankömmlingen gegenwärtig rasch vor sich geht. Da fast jedes Jahr neue Arten hinzukommen, kann diese Gruppe, wie die Autoren der Flora alpina richtig vermerken, sicher nie vollständig erfasst werden. Gerade am Südfuss der Alpen, in der Südwestschweiz und in grossen Städten sind Neophyten wegen des günstigen warmen Klimas und der zahlreichen exotischen Arten in den Gärten und Pärken kaum zu überblicken. Die Anzahl der Taxa könnte wahrscheinlich alle zehn Jahre um mehrere Dutzend vermehrt werden. Zur historischen Dokumentation ist die frühzeitige Erkennung und Kartierung der neuen Einwanderer wichtig. Dazu sollen auch Belege gesammelt und in Herbarien deponiert werden. Die in vielen Gebieten ungenügend bekannte Verbreitung von Neophyten kann durch freiwillige Feldbotaniker abgeklärt werden, die vor allem auf die morphologischen Eigenschaften dieser Arten hinzuweisen sind.

Sich apomiktisch fortpflanzende Taxa sind in vielen Floren nur teilweise aufgeführt, da es ein Spezialwissen braucht, um diese sicher zu kennen. Besonders Kleinarten von Rubus, Alchemilla, Ranunculus auricomus, Festuca, Hieracium, Taraxacum können oft nicht vom gleichen Bearbeiter dargestellt werden. An und für sich wäre es wünschenswert, die Aggregate von Apomikten aufzuspalten, weil Kleinarten oft charakteristische Standorte besiedeln, z. B. bei Taxa von Alchemilla oder vom Ranunculus auricomusAggregat. Gerade in den Alpen haben die apomiktischen Kleinarten oft andere Standortsansprüche als jene des Tieflandes. So ist etwa das Aggregat von R. auricomus L. dort durch wenige charakteristische Kleinarten vertreten, z. B. durch Ranunculus allemannii Br.-Bl. in der subalpinen Stufe der kontinentalen mittleren Alpen. Einzelne Kleinarten, die morphologisch und standörtlich gut erkennbar sind, sollten deshalb auch dargestellt werden, wenn die Mehrzahl der übrigen Kleinarten nicht genau bekannt ist. Mittelfristig ist indessen kaum zu erwarten, dass alle Aggregate apomiktischer Kleinarten vollständig gegliedert werden können.

\section{Ungenügend bearbeitete und fragliche Taxa}

Da auch der beste Taxonom nie alle Taxa eindeutig kennen und bestimmen kann, werden in eine neue Flora oft zahlreiche Sippen aus anderen Florenwerken eingebracht, ohne dass eine ausreichende Überprüfung ihre Eigenständigkeit erfolgt. Umgekehrt werden möglicherweise Taxa nicht aufgenommen, in denen der Autor mangels Erfahrung keine realen Einheiten erkennt. In der Flora alpina werden über 200 fragliche Taxa erwähnt, deren Stellung nicht geklärt ist. Auf der anderen Seite sind aber verschiedene Taxa berücksichtigt worden, deren Eigenständigkeit überprüft werden müsste. Über die Berechtigung vieler Taxa sind sich die Experten nicht einig, wobei nicht immer objektive Beweggründe klar liegen. Deshalb bleibt dann manche Information verschlossen oder in schlecht zugänglichen Quellen versteckt. Fragliche Taxa müssten konsequent zwischen den Kennern diskutiert und klärende Untersuchungen angeregt werden. Einige Beispiele sollen die Probleme näher bringen. 
Die ersten drei Beispiele betreffen Aggregate, von denen nur geographisch oder methodisch begrenzte Untersuchungen oder Beobachtungen vorliegen.

Leontodon hispidus L. s.l. ist ein Aggregat, von dem mir keine Bearbeitung über das gesamte Alpengebiet bekannt ist. Wir können in verschiedenen Gebieten scheinbar klar umschriebene Einheiten feststellen, aber in anderen sind offenbar die Grenzen nicht deutlich. Zwischen den einzelnen Taxa, die soweit wir heute wissen, alle die gleiche Chromosomenzahl 2n=14 besitzen, sind Bastarde möglich, und, wo zwei Taxa zusammen vorkommen, gibt es Übergänge. In der Flora alpina sind keine dieser Taxa angeführt, weil sie offenbar nicht in allen Teilen der Alpen gleich gut zu erkennen sind. So fehlt etwa L. hyoseroides Rchb. (= L. hispidus subsp. hyoseroides) eine charakteristische Sippe kalkhaltiger Schutthänge, die nach Schinz und Thellung (1914) besonders ausgeprägt in den Südalpen, aber auch in anderen Gebieten der mittleren Alpen und im Alpenvorland vorkommt und sich dort morphologisch und standörtlich eindeutig abtrennen lässt.

Chromosomenzählungen weisen auf die besondere zytologische Situation innerhalb eines Aggregates. Ohne ergänzende morphologische und geographische Abklärungen sowie eventuell molekulargenetische Untersuchungen sind aber abschliessende taxonomische Beurteilungen oft nicht möglich. Favarger (1965) hat die Chromosomenrassen des Aggregates von Arenaria ciliata L. beispielhaft zusammengestellt und zum Teil auch morphologisch charakterisiert (Abb. 1). Er konnte dabei 3 Taxa unterscheiden, eine diploide Sippe der äusseren Alpenketten (A. multicaulis L.), eine tetra-, hexa- und octoploide Populationen umfassende inneralpine Sippe (A. ciliata s.str.) und eine dodecaploide Sippe (A. bernensis Favarger). Neben dieser letzteren, die vom Gantrisch und vom Stockhorn bekannt ist, gibt es eine decaploide Sippe vom Wiener Schneeberg, die sich offenbar von der dodecaploiden morphologisch nicht unterscheidet. Gehören nun diese beiden hochploiden Sippen zum gleichen Taxon oder sind sie unabhängig voneinander entstanden und allenfalls als zwei lokal beschränkte Endemiten zu betrachten? Zur Lösung dieser Frage müsste mehr lebendes Material aus dem ganzen Alpengebiet aufgesucht und die wichtigsten Herbarien nachgeprüft werden. Das Fehlen dieser ergänzenden Angaben hatte zur Folge, dass das Taxon A. bernensis heute nur selten in neuere Floren aufgenommen ist.

In ähnlicher Weise werden heute die beiden von Contandriopoulos und Favarger (1959) untersuchten Chromosomensippen von Leucanthemopsis alpina (L.) Heywood s.l. in Floren meist nicht unterschieden, obwohl sie geographisch gut charakterisiert sind (die diploide L. minima (Vill.) Marchi in den westlichen Zentralalpen und die tetraploide L. alpina s.str. in den gesamten Alpen). Morphologische Unterschiede werden aber nur angedeutet.

Bei manchen Taxa sind trotz umfassenden Studien Unklarheiten geblieben, so zum Beispiel beim Aggregat des Aconitum lycoctonum L. Warncke hat 1964 eine Studie über die Gruppe in Europa veröffentlicht. Anhand von Herbarstudien wurden 5 Unterarten beschrieben und breite morphologische Übergangszonen festgestellt. Seine Ergebnisse wurden von späteren Autoren nur teilweise übernommen. In der Flora alpina sind aus dieser Gruppe z. B. zwei Taxa aufgenommen. Mit einer molekulargenetischen Analyse hoffte man mehr Klarheit zu erhalten. Sorgfältige Untersuchungen von Utelli (2005) haben aber im ganzen europäischen Bereich des Aggregates keine deutlichen Gruppierungen aufgezeigt, so dass die Autorin zum Schluss kommt, dass die Gruppe kaum systematisch unterteilt werden kann. Dieses unerwartete Resultat ist gerade für Taxonomen der Alpenflora schwer verständlich. Denn es hätte zur Folge, dass z. B. standörtlich und phänologisch so unterschiedliche Taxa wie 


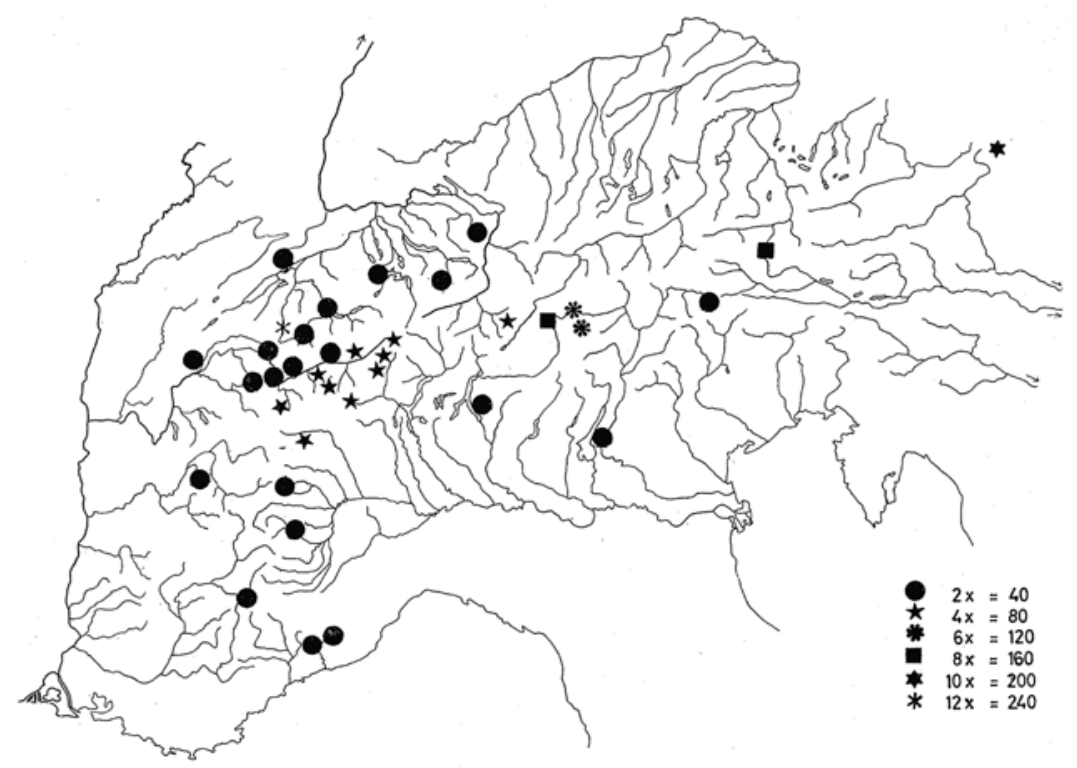

Abb. 1. Verbreitung der Chromosomensippen von Arenaria ciliata aggr. in den Alpen (Favarger 1965).

A. lamarckii Rchb. (= A. ranunculifolium Rchb.) (Kalkgerölle der subalpin-alpinen Stufe) und A. altissimum Mill. (= A. vulparia Rchb.) (Feuchtwälder in der collin-montanen Stufe) systematisch nicht mehr unterschieden werden sollten. Das Beispiel zeigt, dass auch mit modernsten molekularbiologischen Methoden, die sonst die Systematik sehr stark gefördert haben, nicht immer alle taxonomischen Probleme zu lösen sind, bzw., dass es weitere Abklärungen braucht.

Im Laufe der langjährigen Erforschung der Alpenflora sind innerhalb von Aggregaten viele Taxa beschrieben worden, die je nach Autorität der Beschreiber und je nach Erfahrung der Autoren späterer Florenwerke übernommen wurden oder nicht. In vielen Artengruppen ist die Unsicherheit nach über 100 oder 200 Jahren nicht geringer geworden, vor allem deshalb, weil niemand seither diesen Problemen umfassend nachgegangen ist, oder weil zwar Untersuchungen durchgeführt, aber seither übersehen oder als irrelevant beurteilt wurden.

Als Beispiel erwähne ich Alnus brembana, die Rota im 19. Jahrhundert beschrieben hat. Schinz und Thellung (1914) erwähnen das Taxon als Varietät, ebenso Braun-Blanquet und Rübel (1932-1936). In neueren Floren wird es kaum erwähnt, obwohl die Verbreitung und der Standort des Taxons seither abgeklärt wurden (Landolt 1993). Dieses unterscheidet sich morphologisch klar von $A$. viridis und ist auf das insubrische Alpengebiet und das Vorderrheintal beschränkt (Abb. 2). Es besiedelt Silikathänge in südlicher, sonniger Lage. A. viridis ist dem gegenüber eine mittel- und osteuropäische Gebirgspflanze und in den Mittel- und Ostalpen auf nassen Böden vor allem in nördlich exponierten, luftfeuchten Lagen sehr verbreitet. A. brembana ist in allen Teilen kleiner als $A$. viridis (in Klammer die Angaben für $A$. viridis): Höhe $0.8-1.5$ m (1.5- 


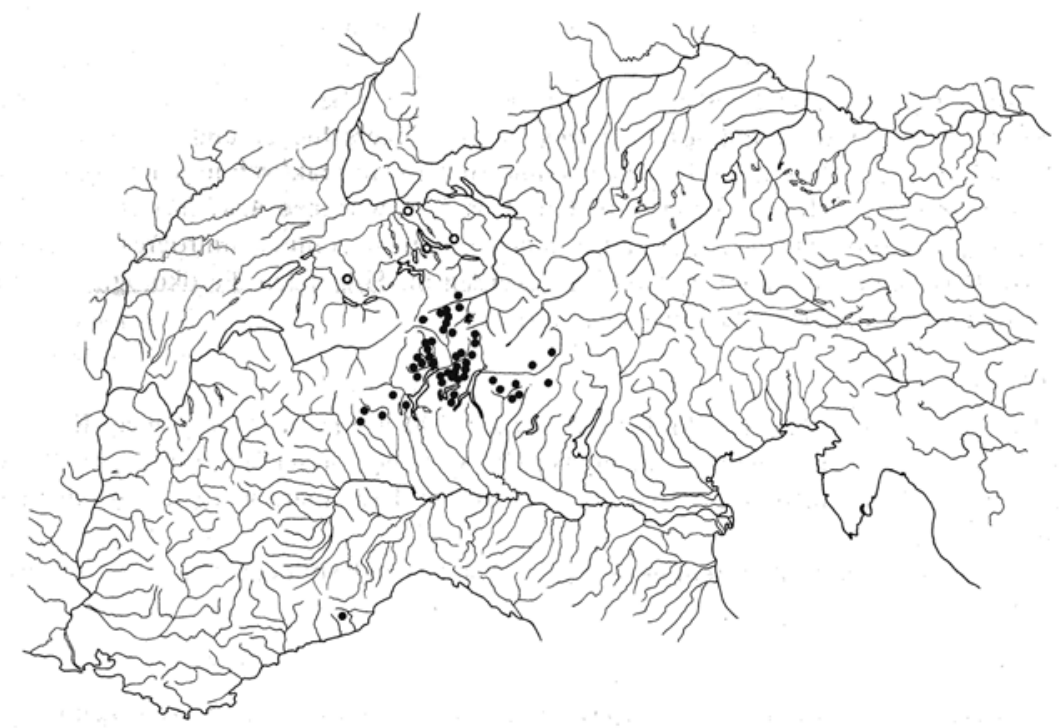

Abb. 2. Verbreitung von Alnus brembana (Landolt 1993). Die leeren Kreise betreffen kurzzeitige adventive Vorkommen, die aus dem 19. Jahrhundert belegt sind.

$3 \mathrm{~m})$, Blattlänge 0.8-3.0 cm (3-6 cm), Länge der männlichen Kätzchen 1-3 cm (3$6 \mathrm{~cm})$, Länge der Zapfen $0.5-0.9 \mathrm{~cm}(0.8-1.5 \mathrm{~cm})$. In Gegenden, wo die beiden Taxa gemeinsam vorkommen, sind breite Zonen von morphologischen Übergangsformen vorhanden. Die Pflanzen von $A$. brembana erscheinen dann bloss als eine Extremform von $A$. viridis. Berücksichtigt man aber die Verbreitung und die Standortsansprüche, so ist das Taxon als selbständige Einheit gut charakterisiert.

Das nächste Beispiel dokumentiert eine Taxonaufspaltung, die wahrscheinlich ungerechtfertigt ist. Bereits 1819 wurde aus Tschechien eine Filipendula denudata J. Presl \& C. Presl von F. ulmaria L. unterschieden. Diese Abtrennung (als Art, Unterart oder Varietät) wird in neueren Floren teilweise wieder aufgenommen (z. B. Flora alpina, Oberdorfer 2001). Die beiden Sippen sind nach der Literatur sehr leicht zu unterscheiden: F. ulmaria s.str. hat weisse (dicht behaarte) Blattunterseiten, F. denudata hellgrüne (fast kahle). In den Herbarien ist meist nur eine Pflanze vom gleichen Fundort vorhanden, die dann eindeutig einem Taxon zugeordnet werden kann. Ich habe in der Umgebung von Zürich und im Alpenvorland viele Populationen untersucht und selbst in kleinen Populationen fast immer beide Erscheinungsformen im Mittel zu etwa gleichen Teilen und ohne Hinweise auf standörtliche Unterschiede gefunden. Daraus könnte man folgern, dass die beiden Formen keine eigenständigen Einheiten bilden und die beiden Merkmale nur durch ganz wenige Gene bewirkt werden. Allerdings ist auch denkbar, dass die vermuteten Taxa nicht gleichmässig über das Alpengebiet verteilt sind und sich an anderen Orten wie zwei getrennte Sippen verhalten. Die beiden Taxa zeigen in der Flora alpina eine unterschiedliche Verbreitung (Band 1, S. 730-732). In den Alpen ist danach $F$. denudata auf die östlichen Teile beschränkt (östlich der 


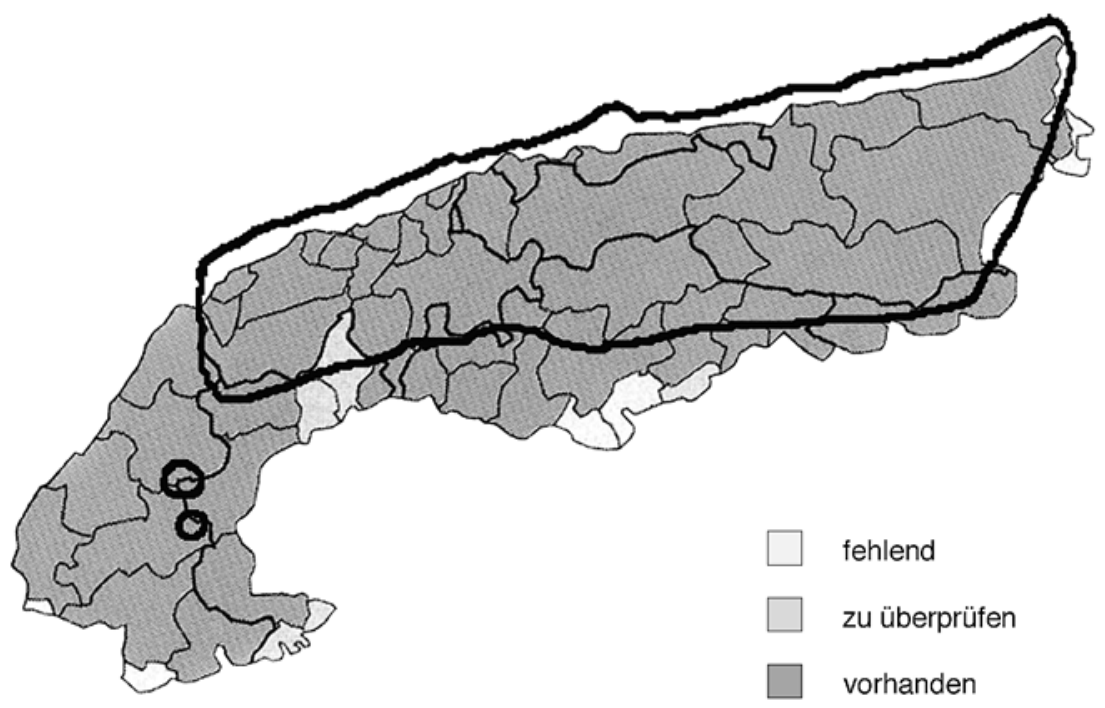

Abb. 3. Verbreitung von Ranunculus montanus s. str. in den Alpen (Aeschimann et al. 2004). Die Verbreitung nach Landolt (1954) ist durch schwarze Umrandungen gekennzeichnet.

Schweizer Grenze), während F. ulmaria s.str. durchgehend im ganzen Alpengebiet vorkommt. Nimmt man aber Schweizer Regionalfloren zur Hand, so werden oft beide Taxa erwähnt. Seitter (1989) schreibt allerdings, dass in Lagen über $1000 \mathrm{~m}$ fast ausschliesslich Pflanzen von F. ulmaria s.str. vorkommen. Unter seinen Fundortsaufzählungen erwähnt er indessen auch $F$. denudata von höheren Lagen. Nach Becherer (1956) sind beide Taxa im Wallis verbreitet. So scheint die Unterscheidung der beiden Sippen $F$. ulmaria und $F$. denudata kaum gerechtfertigt. Wahrscheinlich sind Studien über die Verbreitung der beiden Merkmale schon mehrfach durchgeführt, aber die Ergebnisse nicht publiziert oder weitergegeben worden. Ob die Gliederung von Filipendula sinnvoll ist, müssten weitere, auch von Laien einfach durchführbare Studien über das ganze Alpengebiet zeigen. Die Frage ist, ob es Gegenden gibt, wo nur eine Form auftritt oder wo sich die beiden Formen standörtlich unterscheiden. Entsprechende Resultate müssen später diskutiert und die Folgerungen in der Datenbank des CRSF in Genf festgehalten werden. Sonst besteht die Gefahr, dass diese beiden Formen wie bis anhin auch in neuen Floren nach subjektivem Empfinden als Arten, Unterarten, Varietäten oder gar nicht anerkannt werden.

Dass auch gute Kenner der Alpenflora unmöglich ein genaues Wissen über alle Taxa haben können, zeigt das nächste Beispiel. Die Verbreitung von Ranunculus montanus in den Alpen wurde von mir 1953 anhand von vielen Tausend Herbarexemplaren und auf zahlreichen Exkursionen abgeklärt (Landolt 1954). Die Art fehlt danach in den Südwestalpen weitgehend. Auf der Karte in der Flora alpina ist sie aber selbst in den südlichsten Flächen der Alpen (Haute-Provence, Hautes-Alpes, Alpes-Maritimes, Drôme) sowie in den Apenninen eingezeichnet (Abb. 3). Die Angaben für diese und weitere Flächen gehen wahrscheinlich auf ältere Floren zurück, in denen die Art aus 


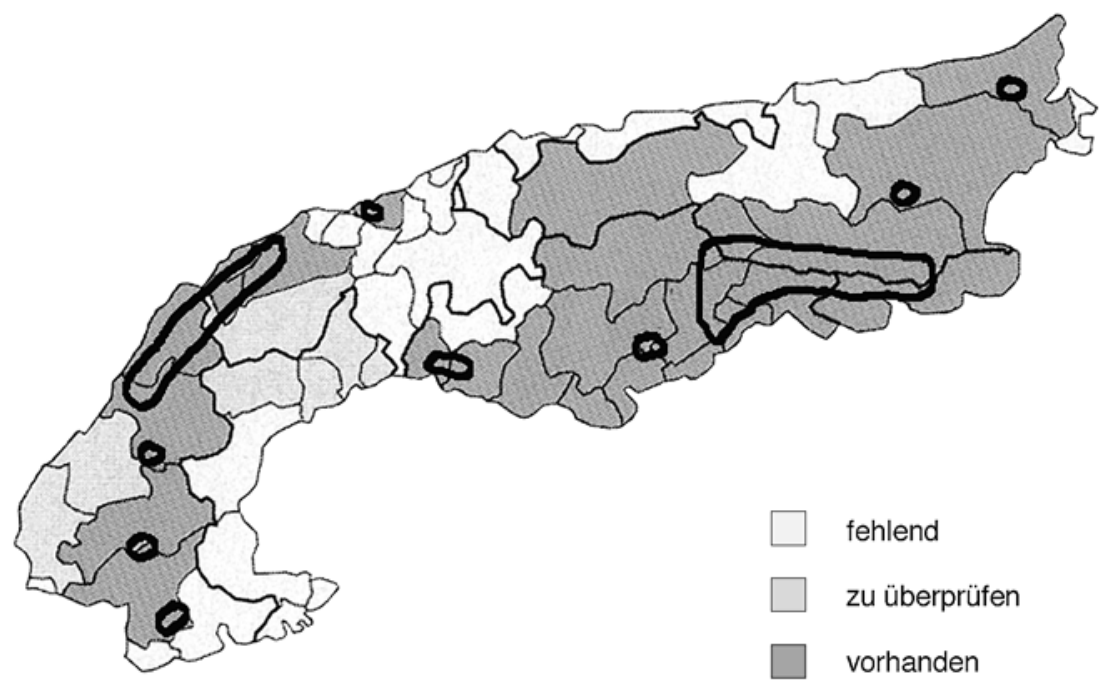

Abb. 4. Verbreitung von Ranunculus carinthiacus in den Alpen nach Aeschimann et al. (2004). Die Verbreitung nach Landolt (1954) ist durch schwarze Umrandungen gekennzeichnet.

diesen Gebieten angegeben, aber nicht vom dort wachsenden nah verwandten $R$. villarsii unterschieden wird.

\section{Kenntnis und Darstellung der Verbreitung}

Die Kenntnis der Verbreitung von Taxa gibt Auskunft über deren Geschichte, Überdauerungs- und Ausbreitungsmöglichkeiten, Variationen der Standortansprüche usw. Wünschbar, aber kurzfristig zu aufwändig, wäre eine Punktkarte für sämtliche eigenständigen Taxa. Auch Verbreitungskarten wie sie für die floristische Kartierung der Schweiz verwendet wurden (Welten und Sutter 1982), d.h. auf der Basis von in der Grösse vergleichbaren Flächen von etwa $100 \mathrm{~km}^{2}$ geben ein aussagekräftiges Bild. Werden die Flächen aber im Verhältnis zur Arealgrösse der Art zu gross oder nehmen sie ungleiche Grössen ein, führen Karten zu falschen oder wenig aussagekräftigen Darstellungen.

Die Angaben in der Flora alpina, die zum ersten Mal Verbreitungskarten zu jeder Art in den Alpen vermittelt, beruhen auf politischen Regionen von sehr unterschiedlicher Grösse. So misst die Steiermark $16400 \mathrm{~km}^{2}$, Liechtenstein aber nur $160 \mathrm{~km}^{2}$, also ein Verhältnis von rund hundert zu eins. Für verbreitete Arten spielt dieser Unterschied keine grosse Rolle, für seltene Arten gibt er oft einen verzerrten Eindruck.

Wie unterschiedlich die Verbreitung der in der Flora alpina angewandten Flächendarstellung mit jener einer zusammengefassten Punktkarte ist, zeigt das Beispiel von Ranunculus carinthiacus (Abb. 4). Die schwarze Umrandung der Punkte aus der Karte von Landolt (1954) ergibt ein differenziertes Verbreitungsgebiet einer disjunkt vor- 
kommenden Art, die grösstenteils in ehemals unvergletscherten Teilen der äusseren Kalkalpen wächst. Nach der Karte in der Flora alpina erscheint die Art in den Alpen sehr weit und flächig verbreitet.

Ähnlich liegen die Verhältnisse bei Valeriana saliunca. Die Flora alpina zeigt zwei grössere Verbreitungsgebiete, die durch einen breiten Korridor in der Schweiz getrennt sind. Nach Merxmüller (1952) ist aber die westalpine Art nur an fünf isolierten Fundstellen östlich der Furka vorhanden.

Auch für Neophyten kann eine Flächendarstellung falsche Vorstellungen erwecken. So scheint die Zeder (Cedrus atlantica) nach der Karte in der Flora alpina flächenmässig etwa über einen Viertel der Alpen verbreitet und erweckt den Anschein, dass sie bereits in weiten Teilen der Alpen eingebürgert ist. Tatsächlich dürfte sie indessen bis heute kaum viel mehr als ein Flächenprozent bewachsen (vor allem in den Südwestalpen). Die übrigen Angaben betreffen wohl seltene lokale Verwilderungen in der Nähe von Kulturen.

Oft ist allerdings nach der Literatur oder nach Angaben von Feldbotanikern die Kenntnis der tatsächlichen Verbreitung einzelner Taxa besser, als dies die Flora alpina zeigt. Hier wird vor allem für kritische Arten und geographisch für die Schweiz die Verbreitung oft als „noch zu ergänzen“ angegeben. Dies geschah aus der Vorsicht, dass schwierige Arten nicht immer richtig erkannt werden und deren Verbreitungsangaben deshalb unzuverlässig sein könnten. Indessen gibt es aber Angaben in der Literatur, auf die man sich verlassen kann und die in die Datenbank aufgenommen werden müssen, auch wenn die Arten in Welten und Sutter (1982) nicht berücksichtigt wurden.

Als Beispiel diene die Artengruppe der Campanula patula. C. costae wird in der Flora alpina aus der Schweiz nur vom Wallis mit Sicherheit angegeben. Hauser (1975) hat in ihrer monographischen Bearbeitung der Gruppe die Verbreitung dieser Sippen in den mittleren und östlichen Alpen genau abgeklärt (zur Namengebung siehe Landolt 1975): C. costae kommt in folgenden Kantonen vor: Wallis, Tessin und Graubünden, aber auch in den Provinzen Como und Torino. C. patula s.str. fehlt dagegen dem Tessin und den meisten südlich der Alpen gelegenen Gebieten.

\section{Vorschläge zur Verbesserung unserer Kenntnisse}

Die heutigen Kenntnisse der Alpenflora und die wünschbaren Verbesserungen können wie folgt zusammengefasst werden:

1. Eine konsequente Übersicht über die Probleme der Taxonomie von Aggregaten in den Alpen fehlt; es braucht deshalb langfristig eine ergänzende kritische Flora der Alpen, in der alle Probleme und Unsicherheiten angeführt sind und auf Literatur verwiesen wird; möglichst viele Spezialisten für kritische Aggregate sind bei der Ausarbeitung beizuziehen und allfällige Interpretationsdifferenzen festzuhalten.

2. Viele Artengruppen sind heute taxonomisch nicht oder nur ungenügend erforscht; oft sind lokale oder regionale Bearbeitungen vorhanden, die auf das ganze Alpengebiet ausgedehnt werden müssen; vorhandene Untersuchungen einzelner Aspekte von Aggregaten sind umfassend zu erweitern. Besonderes Gewicht ist auf die vergleichende morphologische Kenntnis zu legen. Schwierige Aggregate sind durch Experten oder als Doktorarbeiten und mit den neuesten Methoden zu bearbeiten. 
3. Zahlreiche ältere relevante Angaben stehen in oft schwierig erhältlichen Publikationen und sind in Datenbanken nicht vorhanden. Sie werden deshalb leicht übersehen oder vergessen. Diese ältere Literatur muss von Experten kritisch ausgewertet und in die in Genf bestehende Datenbank des CRSF aufgenommen werden.

4. Viele kleinere Probleme könnten auch durch Laienbotaniker abgeklärt werden, sofern sie Anleitung erhalten: oftmals besitzen diese ein einzigartiges Wissen über einzelne Taxa und Gegenden, das unbedingt genutzt werden sollte.

5. Die Verbreitungsangaben sind von den politisch umgrenzten Grossarealen zu lösen; eine Punktkarte ist aufwändig und mittelfristig kaum finanzierbar; es sind deshalb die mutmasslichen Areale oder Teilareale zu umgrenzen. Dazu sollen die bereits zum Teil vorhandenen Unterlagen in Regionalfloren und Verbreitungsatlanten kritisch berücksichtigt werden.

Für Diskussionen und Hinweise danke ich Sabine Güswell, David Aeschimann, Rolf Rutishauser, einem anonymen Begutachter und Walter Lämmler, dem ich auch für die Gestaltung der Abbildungen verbunden bin.

\section{References}

Aeschimann D. und Heitz Ch. 2005. Synonymie-Index der Schweizer Flora. 2. Aufl. Doc. Floristicae Helvetiae 2: 1-317.

Aeschimann D., Lauber K., Moser D. M. und Theurillat J.-P. 2004. Flora alpina. 3 Bde. Haupt, Bern.

Becherer A. 1956. Florae Vallesiacae Supplementum. Denkschr. Schweiz. Naturforsch. Ges. 81: 1-556.

Braun-Blanquet J. und Rübel E. 1932-1936. Flora von Graubünden 1-4. Veröff. Geobot. Inst. Rübel, Zürich 7.

Contandriopoulos J. et Favarger C. 1959. Existence des races chromosomiques chez Chrysanthemum alpinum L. Leur répartition dans les Alpes. Revue Gen. Bot. 66: 341-358.

Favarger C. 1965. A striking polyploid complex in the alpine flora: Arenaria ciliata L. Botaniska Notiser 118: 273-280.

Grossmann F. 1975. Morphologisch-ökologische Untersuchungen an Scabiosa columbaria L. s. 1. im mittleren und westlichen Alpengebiet. Veröff. Geobot. Inst. ETH, Stiftung Rübel 52: 1-125.

Hauser M. L. 1975. Zytotaxonomische Untersuchungen an Campanula patula L. s. 1. und $C$. rapunculus in der Schweiz und in Österreich. Veröff. Geobot. Inst. ETH, Stiftung Rübel 53: $1-70$.

Kremer P. 2004. Phytodiversität in Deutschland. Nat.wiss. Rundsch. 674: 452-454.

Landolt E. 1954. Die Artengruppe des Ranunculus montanus Willd. in den Alpen und im Jura (zytologisch-systematische Untersuchungen). Ber. Schweiz. Bot. Ges. 64: 9-83.

Landolt E. 1975. Nomenklatorische Bemerkungen zur Publikation von M. Hauser über die Artengruppe der Campanula patula L. Veröff. Geobot. Inst. ETH, Stiftung Rübel 53: 71-73.

Landolt E. 1993. Die systematische und pflanzensoziologische Stellung von Alnus brembana (Betulaceae) in den Südalpen. Fragm. Flor. Geobot. Suppl. 2: 521-537.

Merxmüller H. 1952. Untersuchungen zur Sippengliederung und Arealbildung in den Alpen. Bot. Staatsslg. München.

Moser D.M., Gygax A. und Bäumler B. 2005. Fortschritte in der Floristik der Schweizer Flora (Gefässpflanzen). 70. Folge. Bot. Helv. 115: 178-191.

Oberdorfer E. 2001. Pflanzensoziologische Exkursionsflora für Deutschland und angrenzende Gebiete. 8. Aufl. Ulmer, Stuttgart. 
Schinz H. und Thellung A. 1914. Flora der Schweiz, 2. Teil: Kritische Flora. 3. Aufl. Raustein, Zürich.

Seitter H. 1989. Flora der Kantone St. Gallen und beider Appenzell. 2 Bde. St. Galler Naturw. Ges. Kantonaler Lehrmittelverlag, Rorschach.

Utelli A.-B. 2005. Genetic variation, mating system, and nectar robbery in Aconitum lycoctonum (Ranunculaceae). Diss. ETH Zürich.

Warncke K. 1964. Die europäischen Sippen der Aconitum lycoctonum-Gruppe. Diss. Uni München.

Welten M. und Sutter R. 1982. Verbreitungsatlas der Farn- und Blütenpflanzen der Schweiz. 2 Bde. Birkhäuser, Basel. 